\title{
Generalized Wintgen inequality for BI-SLANT submanifolds in conformal Sasakian space form with quarter-symmetric connection
}

\author{
Mohd Aslam \\ Department of Mathematics, Faculty of Natural Sciences, Jamia Millia Islamia, \\ New Delhi, India \\ Mohd Danish Siddiqi \\ Department of Mathematics, Jazan University, Jazan, Saudi Arabia, and \\ Aliya Naaz Siddiqui \\ Department of Mathematics, Maharishi Markandeshwar (Deemed to be University), \\ Mullana-Ambala, India
}

\begin{abstract}
Purpose - In 1979, P. Wintgen obtained a basic relationship between the extrinsic normal curvature the intrinsic Gauss curvature, and squared mean curvature of any surface in a Euclidean 4-space with the equality holding if and only if the curvature ellipse is a circle. In 1999, P. J. De Smet, F. Dillen, L. Verstraelen and L. Vrancken gave a conjecture of Wintgen inequality, named as the DDVV-conjecture, for general Riemannian submanifolds in real space forms. Later on, this conjecture was proven to be true by Z. Lu and by Ge and Z. Tang independently. Since then, the study of Wintgen's inequalities and Wintgen ideal submanifolds has attracted many researchers, and a lot of interesting results have been found during the last 15 years. The main purpose of this paper is to extend this conjecture of Wintgen inequality for bi-slant submanifold in conformal Sasakian space form endowed with a quarter symmetric metric connection.

Design/methodology/approach - The authors used standard technique for obtaining generalized Wintgen inequality for bi-slant submanifold in conformal Sasakian space form endowed with a quarter symmetric metric connection.

Findings - The authors establish the generalized Wintgen inequality for bi-slant submanifold in conformal Sasakian space form endowed with a quarter symmetric metric connection, and also find conditions under which the equality holds. Some particular cases are also stated.

Originality/value - The research may be a challenge for new developments focused on new relationships in terms of various invariants, for different types of submanifolds in that ambient space with several connections.

Keywords Normalized scalar curvature, Scalar curvature, Mean curvature, Quarter-symmetric connection, Conformal Sasakian space form
\end{abstract}

Paper type Research paper

JEL Classification - 34B10, 34B15

(C) Mohd Aslam, Mohd Danish Siddiqi and Aliya Naaz Siddiqui. Published in Arab Journal of Mathematical Sciences. Published by Emerald Publishing Limited. This article is published under the Creative Commons Attribution (CC BY 4.0) license. Anyone may reproduce, distribute, translate and create derivative works of this article (for both commercial and non-commercial purposes), subject to full attribution to the original publication and authors. The full terms of this license may be seen at http:// creativecommons.org/licences/by/4.0/legalcode

The authors are grateful to the referees for the valuable suggestions and comments toward the improvement of the paper.

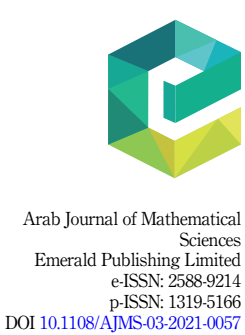




\section{Introduction}

In 1980, I. Vaisman [1] introduced the concept of conformal changes (or deformation) of almost contact metric structures as follows: Let $\tilde{\mathcal{M}}$ be a $(2 n+1)$-dimensional manifold endowed with an almost contact metric structure $(\varphi, \xi, \eta, g)$. A conformal change of the metric $g$ leads to a metric which is no more compatible with the almost contact structure $(\varphi, \xi, \eta)$. This can be corrected by a convenient change of $\xi$ and $\eta$ which implies rather strong restrictions. Using this definition, a new type of almost contact metric structure $(\varphi, \xi, \eta, g)$ on a $(2 n+1)$-dimensional manifold $\tilde{\mathcal{M}}$ which is said to be a conformal Sasakian structure if the structure $(\varphi, \xi, \eta, g)$ is conformal related to a Sasakian structure $(\tilde{\varphi}, \tilde{\xi}, \tilde{\eta}, \tilde{g})$.

The Wintgen inequality is a sharp geometric inequality for surfaces in four-dimensional Euclidean space involving Gauss curvature (intrinsic invariants), normal curvature and square mean curvature (extrinsic invariants). P. Wintgen [2] proved that the Gauss curvature $K$, the normal curvature $K^{\perp}$ and the squared mean curvature $\|\mathcal{H}\|^{2}$ for any surface $\tilde{\mathcal{M}}^{2}$ in $\mathbb{E}^{4}$ satisfy the inequality [3] as follows:

$$
\|\mathcal{H}\|^{2} \geq K+\left|K^{\perp}\right|
$$

and the equality holds if and only if the ellipse of curvature of $\tilde{\mathcal{M}}^{2}$ in $\mathbb{E}^{4}$ is a circle. Later, it was extended by I. V. Gaudalupe et al. [4] for arbitrary codimension $m$ in real space forms $\tilde{\mathcal{M}}^{(m+2)}(c)$ as follows:

$$
\|\mathcal{H}\|^{2}+c \geq K+\left|K^{\perp}\right| .
$$

In 1999, De Smet, Dillen, Verstraelen and Vrancken [5] conjectured the generalized Wintgen inequality for submanifolds in real space form. The conjecture is known as DDVV conjecture. It had been proved by Lu [6] and by Ge and Tang [7] independently. In 2014, Ion Mihai [8] established such inequality for Lagrangian submanifold in complex space form. They provided some applications and also stated such an inequality for slant submanifolds in complex space forms. However, the year 2014 is not the stopping point in investigating Wintgen inequality and some additional steps have been taken in the development of the theory. In fact, many remarkable articles were published in the recent years and several inequalities of this type have been obtained for other classes of submanifolds in several ambient spaces for example, for statistical submanifolds in statistical manifolds of constant curvature [9]; for Legendrian submanifolds in Sasakian space forms [10]; for submanifolds in statistical warped product manifolds [11]; for quaternionic CR-submanifolds in quaternionic space forms [12]; for submanifolds in generalized $(\kappa, \mu)$-space forms [13]; for totally real submanifolds in LCS-manifolds [14] and so on. For more details, see [15].

In the present article, we obtain the generalized Wintgen inequalities for conformal Sasakian space forms. The equality case of the main inequality is investigated. Lastly, we discuss such inequality for various slant cases as an application of the obtained inequality.

\section{Preliminaries}

\subsection{Sasakian manifold}

An odd-dimensional Riemannian manifold $(\tilde{\mathcal{M}}, g)$ is said to be an almost contact metric manifold [16] if there exist a tensor $\varphi$ of type $(1,1)$, a vector field $\xi$ (structure vector field) and a 1-form $\eta$ on $\tilde{\mathcal{M}}$ satisfying

$$
\begin{gathered}
\varphi^{2} X=-X+\eta(X) \xi, \quad \eta(\xi)=1, \\
\varphi \xi=0, \quad \eta^{\circ} \xi=0, \quad g(X, \xi)=\eta(X),
\end{gathered}
$$


and

$$
g(\varphi X, \varphi Y)=g(X, Y)-\eta(X) \eta(Y)
$$

for any $X, Y \in \Gamma(T \tilde{\mathcal{M}})$. The two-form $\Phi$ is called the fundamental two-form in $\tilde{\mathcal{M}}$ and the manifold is said to be a contact metric manifold if

$$
\Phi=d \eta
$$

A Sasakian manifold is a normal contact metric manifold. In fact, an almost contact metric manifold is Sasakian manifold if and only if we have

$$
\left(\bar{\nabla}_{X} \varphi\right) Y=g(X, Y) \xi-\eta(Y) X
$$

for any $X, Y \in \Gamma(T \tilde{\mathcal{M}})$, where $\bar{\nabla}$ denotes the Riemannian connection.

A plane section $\pi$ in $T_{p} \tilde{\mathcal{M}}$ is called a $\varphi$-section if it is spanned by $X$ and $\varphi X$, where $X$ is a unit tangent vector orthogonal to $\xi$. The sectional curvature of a $\varphi$-section is called a $\varphi$-sectional curvature. A Sasakian manifold with constant $\varphi$-sectional curvature $c$ is said to be a Sasakian space form and denoted by $\tilde{\mathcal{M}}(c)$. The curvature tensor of a Sasakian space form $\tilde{\mathcal{M}}(c)$ is given by [16].

$$
\begin{aligned}
R(X, Y) Z= & \frac{(c+3)}{4}\{g(Y, Z) X-g(X, Z) Y\}+\frac{(c-1)}{4} \eta(Z) \eta(Y) X \\
& -\eta(X) Y+(g(Y, Z) \eta(X)-g(X, Z) \eta(Y)) \xi-g(\varphi Y, Z) \varphi X \\
& +g(\varphi X, Z) \varphi Y+2 g(\varphi X, Y) \varphi Z,
\end{aligned}
$$

for any $X, Y, Z \in \Gamma(T \tilde{\mathcal{M}})$.

\subsection{Conformal Sasakian manifold}

A $(2 n+1)$-dimensional Riemannian manifold $\tilde{\mathcal{M}}$ endowed with the almost contact metric structure $(\varphi, \eta, \xi, g)$ called a conformal Sasakian manifold if for a $C^{\infty}$ function

$$
f: \tilde{\mathcal{M}} \rightarrow \mathbb{R}
$$

there exists [3].

$$
\begin{gathered}
\tilde{g}=\exp (f) g, \quad \tilde{\xi}=(\exp (-f))^{\frac{1}{2}} \xi \\
\tilde{\eta}=(\exp (f))^{\frac{1}{2}} \eta, \quad \tilde{\varphi}=\varphi
\end{gathered}
$$

such that $(\tilde{\mathcal{M}}, \tilde{\varphi}, \tilde{\xi}, \tilde{g})$ is a Sasakian manifold.

Let $\tilde{\nabla}$ and $\bar{\nabla}$ denote connections of $\tilde{\mathcal{M}}$ related to metrics $\tilde{g}$ and $g$, respectively. Using Koszul formula, we derive the following relation between the connections $\tilde{\nabla}$ and $\bar{\nabla}$ :

$$
\tilde{\nabla}_{X} Y=\bar{\nabla}_{X} Y+\frac{1}{2}\{\omega(X) Y+\omega(Y) X-g(X, Y) \omega\},
$$

for any $X, Y \in \Gamma(\tilde{\mathcal{M}})$ so that $\omega(X)=X(f)$ and $\omega^{\#}$ is vector field of metrically equivalent to one form of $\omega$, i.e. $g\left(\omega^{\#}, X\right)=\omega(X)$. The vector field $\omega^{\#}=\operatorname{grad} f$ is called the Lee vector field of conformal Sasakian manifold $\tilde{\mathcal{M}}$.

The $(2 n+1)$-dimensional conformal Sasakian manifold with constant sectional curvature $c$, denoted by $\tilde{\mathcal{M}}(c)$, is called a conformal Sasakian space form and its curvature tensor is given by [3]. 
AJMS

$$
\begin{aligned}
g(\overline{\mathcal{R}}(X, Y) Z, W)= & \exp (f)\left\{\frac{c+3}{4}(g(Y, Z) g(X, W)-g(X, Z) g(Y, W))\right. \\
& +\frac{c-1}{4}(\eta(X) \eta(Z) g(Y, W)-\eta(Y) \eta(Z) g(X, W) \\
& +g(X, Z) g(\xi, W) \eta(Y)-g(Y, Z) g(\xi, W) \eta(X) \\
& -g(\varphi Y, Z) g(\varphi X, W)-g(\varphi X, Z) g(\varphi Y, W) \\
& -2 g(\varphi X, Y) g(\varphi Z, W))\}-\frac{1}{2}(B(X, Z) g(Y, W) \\
& -B(Y, Z) g(X, W)+B(Y, W) g(Y, Z)-B(X, W) g(Y, Z)) \\
& -\frac{1}{4}\|\omega\|^{2}(g(X, Z) g(Y, W)-g(X, W) g(Y, Z))
\end{aligned}
$$

for any $X, Y, Z, W, \omega, \xi \in \Gamma(T \tilde{\mathcal{M}})$, where $B:=\bar{\nabla} \omega-\frac{1}{2} \omega \otimes \omega$.

\subsection{Quarter-symmetric metric connection}

Let $\tilde{\mathcal{M}}$ be an $(2 n+1)$-dimensional Riemannian manifold with Riemannian metric $g$ and $\nabla$ be the Levi-Civita connection on $\tilde{\mathcal{M}}$. Let $\tilde{\nabla}$ be a linear connection defined by [17].

$$
\tilde{\bar{\nabla}}_{X} Y=\bar{\nabla}_{X} Y+\Lambda_{1} \lambda(Y) X-\Lambda_{2} g(X, Y) \mathcal{V}
$$

for any $X, Y \in \Gamma(\tilde{\mathcal{M}}), \Lambda_{1}$ and $\Lambda_{2}$ are real constants and $\mathcal{V}$ is the vector field on $\tilde{\mathcal{M}}$ such that $\lambda(X)=g(X, \mathcal{V})$, where $\lambda$ is 1 -form. If $\tilde{\bar{\nabla}} g=0$, then $\tilde{\bar{\nabla}}$ is known as quarter-symmetric metric connection and $\tilde{\bar{\nabla}} g \neq 0$, then $\tilde{\bar{\nabla}}$ is known as quarter-symmetric non-metric connection. Decomposing the vector field $\mathcal{V}$ on $\tilde{\mathcal{M}}$ uniquely into its tangent and normal components $\mathcal{V}^{T}$ and $\mathcal{V}^{\perp}$, respectively.

The special cases of (2.5) can be obtained as follows:

(1) when $\Lambda_{1}=\Lambda_{2}=1$, then the above connection reduces to semi-symmetric metric connection and

(2) when $\Lambda_{1}=1$ and $\Lambda_{2}=0$, then the above connection reduces to semi-symmetric nonmetric connection.

For any $X, Y, Z, W \in \Gamma(T \tilde{\mathcal{M}})$, the curvature tensor with respect to $\tilde{\bar{\nabla}}$ is given by

$$
\tilde{\overline{\mathcal{R}}}(X, Y) Z=\tilde{\bar{\nabla}}_{X} \tilde{\bar{\nabla}}_{Y} Z-\tilde{\bar{\nabla}}_{Y} \tilde{\bar{\nabla}}_{X} Z-\tilde{\bar{\nabla}}_{[X, Y]} Z .
$$

On using (2.5), the curvature tensor (2.6) takes the form [17] as follows:

$$
\begin{aligned}
\tilde{\mathcal{\mathcal { R }}}(X, Y, Z, W)= & \overline{\mathcal{R}}(X, Y, Z, W)+\Lambda_{1} \alpha(X, Z) g(Y, W)-\Lambda_{1} \alpha(Y, Z) g(X, W) \\
& +\Lambda_{2} \alpha(Y, W) g(X, Z)-\Lambda_{2} \alpha(X, W) g(Y, Z)+\Lambda_{2}\left(\Lambda_{1}-\Lambda_{2}\right) \\
& g(X, Z) \beta(Y, W)-\Lambda_{2}\left(\Lambda_{1}-\Lambda_{2}\right) g(Y, Z) \beta(X, W),
\end{aligned}
$$

where $\alpha$ and $\beta$ are (0,2)-tensors and defined as follows:

$$
\alpha(X, Y)=\left(\bar{\nabla}_{X} \lambda\right)(Y)-\Lambda_{1} \lambda(X) \lambda(Y)+\frac{\Lambda_{2}}{2} g(X, Y) \lambda(\mathcal{V}),
$$


and

$$
\beta(X, Y)=\frac{\lambda(\mathcal{V})}{2} g(X, Y)+\lambda(X) \lambda(Y)
$$

The curvature tensor of conformal Saasakian space form $\tilde{\mathcal{M}}(c)$ with a quarter-symmetric connection $\tilde{\bar{\nabla}}$ is given by

$$
\begin{aligned}
g(\tilde{\mathcal{\mathcal { R }}}(X, Y) Z, W)= & \exp (f)\left\{\frac{c+3}{4}(g(Y, Z) g(X, W)-g(X, Z) g(Y, W))\right. \\
& +\frac{c-1}{4}(\eta(X) \eta(Z) g(Y, W)-\eta(Y) \eta(Z) g(X, W) \\
& +g(X, Z) g(\xi, W) \eta(Y)-g(Y, Z) g(\xi, W) \eta(X) \\
& -g(\varphi Y, Z) g(\varphi X, W)-g(\varphi X, Z) g(\varphi Y, W) \\
& -2 g(\varphi X, Y) g(\varphi Z, W))-\frac{1}{2}(B(X, Z) g(Y, W) \\
& -B(Y, Z) g(X, W)+B(Y, W) g(Y, Z)-B(X, W) g(Y, Z)) \\
& -\frac{1}{4}\|\omega\|^{2}(g(X, Z) g(Y, W)-g(X, W) g(Y, Z)) \\
& +\Lambda_{1} \alpha(X, Z) g(Y, W)-\Lambda_{1} \alpha(Y, Z) g(X, W) \\
& +\Lambda_{2} g(X, Z) \alpha(Y, W)-\Lambda_{2} g(Y, Z) \alpha(X, W) \\
& +\Lambda_{2}\left(\Lambda_{1}-\Lambda_{2}\right) g(X, Z) \beta(Y, W) \\
& -\Lambda_{2}\left(\Lambda_{1}-\Lambda_{2}\right) g(Y, Z) \beta(X, W) .
\end{aligned}
$$

For simplicity, we have put $\operatorname{tr}(\alpha)=a$ and $\operatorname{tr}(\beta)=b$.

Let $\mathcal{M}$ be an $m$-dimensional submanifold of a $(2 n+1)$-dimensional conformal Saasakian space form $\tilde{\mathcal{M}}(c)$. We consider the induced quarter-symmetric connection on $\mathcal{M}$ represented by $\tilde{\nabla}^{\mathcal{M}}$ and the induced Levi-Civita connection denoted by $\nabla^{\mathcal{M}}$. Let $\mathcal{R}$ and $\mathcal{R}^{\mathcal{M}}$ be the curvature tensors of $\tilde{\nabla}^{\mathcal{M}}$ and $\nabla^{\mathcal{M}}$. Then, the Gauss equation is given by

$$
\begin{aligned}
\tilde{\mathcal{R}}(X, Y, Z, W)= & \mathcal{R}(X, Y, Z, W)-g(h(X, W), h(Y, Z))+g(h(Y, W), h(X, Z)) \\
& +\left(\Lambda_{1}-\Lambda_{2}\right) g\left(h(Y, Z), \mathcal{V}^{\perp}\right) g(X, W) \\
& +\left(\Lambda_{2}-\Lambda_{1}\right) g\left(h(X, Z), \mathcal{V}^{\perp}\right) g(Y, W),
\end{aligned}
$$

where $h$ is the second fundamental form of $\mathcal{M}$ in $\tilde{\mathcal{M}}$ with respect to $\tilde{\bar{\nabla}}$ and defined as follows:

$$
h(X, Y)=h^{\prime}(X, Y)-\Lambda_{2} g(X, Y) \mathcal{V}^{\perp} .
$$

Here, $h^{\prime}$ is the second fundamental form of $\mathcal{M}$ in $\tilde{\mathcal{M}}$ with respect to $\bar{\nabla}$ and $g$ denotes the Riemannian metric on $\mathcal{M}$.

For any $X \in \Gamma(T \mathcal{M})$, we can write $\varphi X=P X+S X$, where the $P X$ (respectively, $S X$ ) is the tangential component (respectively normal component) of $\varphi X$. If $P=0$, then the submanifold is anti-invariant and if $S=0$, then the submanifold is invariant. The squared norm of $P$ at $p \in \mathcal{M}$ is given as follows: 


$$
\|P\|^{2}=\sum_{i, j=1}^{m} g^{2}\left(\varphi e_{i}, e_{j}\right),
$$

where $\left\{e_{1}, \ldots, e_{m}\right\}$ is any orthonormal basis of $T_{p} \mathcal{M}$ and $p \in \mathcal{M}$. The structure vector field $\xi$ can be decomposed as $\xi=\xi^{T}+\xi^{\perp}$, where $\xi^{T}$ and $\xi^{\perp}$ are tangential and normal components of $\xi$.

The notion of bi-slant submanifolds was introduced by A. Carriazo et al. as a natural generalization of CR, slant, semi-slant and hemi-slant submanifolds (see [18-20]). Recently, S. Uddin and B.-Y. Chen studied bi-slant and pointwise bi-slant submanifolds for their warped products in [21, 22]. A submanifold $\mathcal{M}$ of an almost contact-metric manifold $\tilde{\mathcal{M}}$ is called bi-slant submanifolds, whenever we have

(1) $T \mathcal{M}^{m}=\mathcal{D}_{\theta_{1}} \oplus \mathcal{D}_{\theta_{1}} \oplus\langle\xi\rangle$ and

(2) $\varphi \mathcal{D}_{\theta_{1}} \perp \mathcal{D}_{\theta_{2}}$ and $\varphi \mathcal{D}_{\theta_{2}} \perp \mathcal{D}_{\theta_{1}}$

where $\mathcal{D}_{\theta_{1}}$ and $\mathcal{D}_{\theta_{2}}$ are two orthogonal distributions of $\mathcal{M}$ with slant angle $\theta_{1}$ and $\theta_{2}$, respectively.

Let $\mathcal{M}$ be a bi-slant submanifold of a conformal Sasakian space form $\tilde{\mathcal{M}}$. We assume that $\operatorname{dim}(\mathcal{M})=m=2 m_{1}+2 m_{2}+1$, where $\operatorname{dim}\left(\mathcal{D}_{\theta_{1}}\right)=m_{1}$ and $\operatorname{dim}\left(\mathcal{D}_{\theta_{2}}\right)=m_{2}$. Let $\left\{e_{1}, \ldots\right.$, $\left.e_{m}=\xi\right\}$ be an orthonormal basis of $T_{p} \mathcal{M}$ at $p$ in $\mathcal{M}$ with

$e_{1}, e_{2}=\sec \theta_{1} P e_{1}, \ldots, e_{2 m_{1}-1}, e_{2 m_{1}}=\sec \theta_{1} P e_{2 m_{1}-1}, e_{2 m_{1}+1}, e_{2 m_{1}+2}=\sec \theta_{2} P e_{2 m_{1}+1}, \ldots$, $e_{2 m_{1}+2 m_{2}-1}, e_{2 m_{1}+2 m_{2}}=\sec \theta_{2} P e_{2 m_{1}+2 m_{2}-1}, e_{2 m_{1}+2 m_{2}+1}=\boldsymbol{\xi}$,

from which we have [23] as follows:

$g^{2}\left(\varphi e_{i+1}, e_{i}\right)=\left\{\begin{array}{l}\cos ^{2} \theta_{1}, \text { for } i=1,2, \ldots, 2 m_{1}-1 \\ \cos ^{2} \theta_{2}, \text { for } i=2 m_{1}+1, \ldots, 2 m_{1}+2 m_{2}-1 .\end{array}\right.$

Thus, we have

$$
\sum_{i, j=1}^{m} g^{2}\left(\varphi e_{i}, e_{j}\right)=2\left\{m_{1} \cos ^{2} \theta_{1}+m_{2} \cos ^{2} \theta_{2}\right\} .
$$

In fact, semi-slant, pseudo-slant, $\mathrm{CR}$ and slant submanifolds can be obtained from bi-slant submanifolds in particular. We can see the cases in the following Table 1:

The special case of slant submanifold are invariant and anti-invariant if $\theta=0$ and $\theta=\frac{\pi}{2}$, respectively. The slant submanifold is said to be proper slant and proper bi-slant submanifold, if $0<\theta<\frac{\pi}{2}$ and $\theta_{i}$ lies between 0 and $\frac{\pi}{2}$.

Table 1.

Different types of

\begin{tabular}{|c|c|c|c|c|c|}
\hline S N & $\mathcal{M}^{m}$ & $D_{\theta_{1}}$ & $D_{\theta_{2}}$ & $\theta_{1}$ & $\theta_{2}$ \\
\hline (1) & Bi-slant & Slant distribution & Slant distribution & Slant angle & Slant angle \\
\hline (2) & Semi-slant & Invariant distribution & Slant distribution & 0 & Slant angle \\
\hline (3) & Pseudo-slant & Slant distribution & $\begin{array}{l}\text { Anti-invariant } \\
\text { distribution }\end{array}$ & Slant angle & $\frac{\pi}{2}$ \\
\hline (4) & Contact CR & Invariant distribution & $\begin{array}{l}\text { Anti-invariant } \\
\text { distribution }\end{array}$ & 0 & $\frac{\pi}{2}$ \\
\hline (5) & Slant & Either $D_{\theta_{1}}=0$ or $D_{\theta_{2}}=0$ & & $\begin{array}{l}\text { Either } \theta_{1}= \\
\neq \theta\end{array}$ & $\theta$ or $\theta_{1}=\theta_{2}$ \\
\hline
\end{tabular}
submanifolds 
3. Main inequalities

In [10], Mihai discussed the generalized Wintgen inequality for Legendrian submanifolds in Sasakian space forms. He also stated such an inequality for contact slant submanifolds in Sasakian space forms. Thus, in this section, we obtain such an inequality in terms of the invariant $\rho_{\mathcal{M}}$ (called normalized scalar-normal curvature) for bi-slant submanifolds of dimension $m$ in a $(2 n+1)$-dimensional conformal Sasakian space form $\tilde{\mathcal{M}}$. Consider the local orthonormal tangent frame $\left\{e_{1}, \ldots, e_{m}\right\}$ of the tangent bundle $T \mathcal{M}$ of $\mathcal{M}$ and a local orthonormal normal frame $\left\{e_{m+1}, \ldots, e_{2 n+1}\right\}$ of the normal bundle $T^{\perp} \mathcal{M}$ of $\mathcal{M}$ in $\tilde{\mathcal{M}}$. At any $p \in \mathcal{M}$, the scalar curvature $\tau$ at that point is given by

$$
\mathcal{T}=\sum_{1 \leq i<j \leq m} \mathcal{R}\left(e_{i}, e_{j}, e_{j}, e_{i}\right)
$$

The mean curvature $\mathcal{H}$ of submanifold is given by

$$
\mathcal{H}=\frac{1}{m} \sum_{i=1}^{m} h\left(e_{i}, e_{i}\right)
$$

Conveniently, let us put

$$
h_{i j}^{r}=g\left(h\left(e_{i}, e_{i}\right), e_{r}\right),
$$

for any $i, j=\{1, \ldots, m\}$ and $r=\{m+1, \ldots, 2 n+1\}$.

We denote by $K$ and $R^{\perp}$, the sectional curvature function and the normal curvature tensor on $\mathcal{M}$, respectively. Then the normalized scalar curvature $\rho$ is given by [8].

$$
\rho=\frac{2 \tau}{m(m-1)}=\frac{2}{m(m-1)} \sum_{1 \leq i<j \leq m} \kappa\left(e_{i} \wedge e_{j}\right) .
$$

In term of the components of the second fundamental form, we can express the scalar normal curvature $\kappa_{\mathcal{M}}$ of $\mathcal{M}$ by the formula [8].

$$
\kappa_{\mathcal{M}}=\sum_{1 \leq r<s \leq 2 n-m+11 \leq i<j \leq m} \sum_{t=1}\left(\sum_{j t}^{m} h_{i t}^{r} h_{i t}^{s}-h_{j t}^{r} h_{i t}^{s}\right)^{2},
$$

and the normalized scalar normal curvature is given by [8].

$$
\rho_{\mathcal{M}}=\frac{2}{m(m-1)} \sqrt{\kappa_{\mathcal{M}}}
$$

Theorem 3.1. Let $\mathcal{M}$ be an m-dimensional bi-slant submanifold in conformal Sasakian space form $\tilde{\mathcal{M}}(c)$ of dimension $(2 n+1)$ endowed with a quarter-symmetric connection, then we have

$$
\begin{aligned}
\rho_{\mathcal{M}}+\rho & \leq\|\mathcal{H}\|^{2}+\exp (f)\left\{\frac{c+3}{4}-\frac{c-1}{2 m}+\frac{3(c-1)}{4 m(m-1)}\left(m_{1} \cos ^{2} \theta_{1}+m_{2} \cos ^{2} \theta_{2}\right)\right\} \\
& +\frac{\operatorname{tr}(B)}{m}+\frac{1}{4}\|\omega\|^{2}-\left\{\left(\Lambda_{1}+\Lambda_{2}\right) \frac{a}{m}+\Lambda_{2}\left(\Lambda_{1}-\Lambda_{2}\right) \frac{b}{m}-\left(\Lambda_{2}-\Lambda_{1}\right) \lambda(\mathcal{H})\right\} .
\end{aligned}
$$

Generalized

Wintgen inequality 
Moreover, the equality case holds in the above inequality at a point $p \in \mathcal{M}$ if and only if, with respect to some suitable orthonormal basis $\left\{e_{1}, \ldots, e_{m}\right\}$ of $T_{p} \mathcal{M}$ and $\left\{\xi_{1}, \ldots, \xi_{2 n-m+1}\right\}$ of $T_{p}^{\perp} \mathcal{M}$, the shape operators $S_{\gamma}, \gamma=1, \ldots, 2 \mathrm{n}-m+1$, take the forms as follows:

$$
\begin{aligned}
& S_{1}=\left(\begin{array}{ccccc}
\mu_{1} & \zeta & 0 & \ldots & 0 \\
\zeta & \mu_{1} & 0 & \ldots & 0 \\
0 & 0 & \mu_{1} & \ldots & 0 \\
\vdots & \vdots & \vdots & \ddots & \vdots \\
0 & 0 & 0 & \ldots & \mu_{1}
\end{array}\right) \\
& S_{2}=\left(\begin{array}{ccccc}
\mu_{2}+\zeta & 0 & 0 & \ldots & 0 \\
0 & \mu_{2}-\zeta & 0 & \ldots & 0 \\
0 & 0 & \mu_{2} & \ldots & 0 \\
\vdots & \vdots & \vdots & \ddots & \vdots \\
0 & 0 & 0 & \ldots & \mu_{2}
\end{array}\right) \\
& S_{3}=\left(\begin{array}{ccccc}
\mu_{3} & 0 & 0 & \ldots & 0 \\
0 & \mu_{3} & 0 & \ldots & 0 \\
0 & 0 & \mu_{3} & \ldots & 0 \\
\vdots & \vdots & \vdots & \ddots & \vdots \\
0 & 0 & 0 & \ldots & \mu_{3}
\end{array}\right), \quad S_{4}=\cdots=S_{2 n-m+1}=0,
\end{aligned}
$$

where $\mu_{1}, \mu_{2}, \mu_{3}$, and $\zeta$ are real numbers.

Proof. Let $\left\{e_{1}, \ldots, e_{m}\right\}$ and $\left\{e_{m+1}, \ldots, e_{2 n+1}\right\}$ be orthonormal tangent frame and orthonormal normal frame on $\mathcal{M}$, respectively. Putting $X=W=e_{i}, Y=Z=e_{j}, i \neq j$ in (2.9) and using (2.8), we obtain

$$
\begin{aligned}
\tilde{\mathcal{\mathcal { R }}}\left(e_{i}, e_{j}, e_{j}, e_{i}\right)= & \exp (f)\left\{\frac{c+3}{4}\left(g\left(e_{j}, e_{j}\right) g\left(e_{i}, e_{i}\right)-g\left(e_{i}, e_{j}\right) g\left(e_{j}, e_{i}\right)\right)\right. \\
& +\frac{c-1}{4}\left(\eta\left(e_{i}\right) \eta\left(e_{j}\right) g\left(e_{j}, e_{i}\right)-\eta\left(e_{j}\right) \eta\left(e_{j}\right) g\left(e_{i}, e_{i}\right)\right. \\
& +g\left(e_{i}, e_{j}\right) g\left(\xi, e_{i}\right) \eta\left(e_{j}\right)-g\left(e_{j}, e_{j}\right) g\left(\xi, e_{i}\right) \eta\left(e_{i}\right) \\
& -g\left(\varphi e_{j}, e_{j}\right) g\left(\varphi e_{i}, e_{i}\right)-g\left(\varphi e_{i}, e_{j}\right) g\left(\varphi e_{j}, e_{i}\right) \\
& \left.-2 g\left(\varphi e_{i}, e_{j}\right) g\left(\varphi e_{j}, e_{i}\right)\right\}-\frac{1}{2}\left(B\left(e_{i}, e_{j}\right) g\left(e_{j}, e_{i}\right)\right. \\
& \left.-B\left(e_{j}, e_{j}\right) g\left(e_{i}, e_{i}\right)+B\left(e_{j}, e_{i}\right) g\left(e_{i}, e_{j}\right)-B\left(e_{i}, e_{i}\right) g\left(e_{j}, e_{j}\right)\right) \\
& -\frac{1}{4}\|\omega\|^{2}\left(g\left(e_{i}, e_{j}\right) g\left(e_{j}, e_{i}\right)-g\left(e_{j}, e_{j}\right) g\left(e_{i}, e_{i}\right)\right) \\
& +\Lambda_{1} \alpha\left(e_{i}, e_{j}\right) g\left(e_{j}, e_{i}\right)-\Lambda_{1} \alpha\left(e_{j}, e_{j}\right) g\left(e_{i}, e_{i}\right) \\
& +\Lambda_{2} g\left(e_{i}, e_{j}\right) \alpha\left(e_{j}, e_{i}\right)-\Lambda_{2} g\left(e_{j}, e_{j}\right) \alpha\left(e_{i}, e_{i}\right) \\
& +\Lambda_{2}\left(\Lambda_{1}-\Lambda_{2}\right) g\left(e_{i}, e_{j}\right) \beta\left(e_{j}, e_{i}\right)-\Lambda_{2}\left(\Lambda_{1}-\Lambda_{2}\right) g\left(e_{j}, e_{j}\right) \beta\left(e_{i}, e_{i}\right), \\
& -\left(\Lambda_{1}-\Lambda_{2}\right) g\left(h\left(e_{j}, e_{j}\right), \mathcal{P}^{\perp}\right) g\left(e_{i}, e_{i}\right) \\
& -\left(\Lambda_{2}-\Lambda_{1}\right) g\left(h\left(e_{i}, e_{j}\right), \mathcal{P}^{\perp}\right) g\left(e_{j}, e_{i}\right) .
\end{aligned}
$$


By taking summation $1 \leq i<j \leq m$ of (3.9) and using (2.7), we have

$$
\begin{aligned}
\sum_{1 \leq i<j \leq m} \mathcal{R}\left(e_{i}, e_{j}, e_{j}, e_{i}\right)= & \frac{\exp (f)}{2}\left\{\frac{(c+3) m(m-1)}{4}+\frac{(c-1)}{4}\left(2-2 m+3\|P\|^{2}\right\}+\frac{(m-1)}{2} \operatorname{tr}(B)\right. \\
& +\frac{1}{8} m(m-1)\|\omega\|^{2}+\frac{1}{2}\left\{\left(\Lambda_{1}+\Lambda_{2}\right)(1-m) a+\Lambda_{2}\left(\Lambda_{1}-\Lambda_{2}\right)(1-m) b\right. \\
& \left.+\left(\Lambda_{2}-\Lambda_{1}\right) m(m-1) \lambda(\mathcal{H})\right\}+\sum_{r=1}^{2 n-m+1} \sum_{1 \leq i<j \leq m}\left[h_{i i}^{r} h_{j j}^{r}-\left(h_{i j}^{r}\right)^{2}\right],
\end{aligned}
$$

where

$$
\lambda(\mathcal{H})=\frac{1}{m} \sum_{j=1}^{m} \lambda\left(h\left(e_{j}, e_{j}\right)\right)=g\left(\mathcal{V}^{\perp}, \mathcal{H}\right) .
$$

Using (2.10) and (3.1), we obtain

$$
\begin{aligned}
\tau= & \frac{\exp (f)}{2}\left\{\frac{(c+3) m(m-1)}{4}+\frac{(c-1)}{4} 2-2 m+3\left(m_{1} \cos ^{2} \theta_{1}+m_{2} \cos ^{2} \theta_{2}\right)\right\}+\frac{(m-1)}{2} \operatorname{tr}(B) \\
& +\frac{1}{8} m(m-1)\|\omega\|^{2}+\frac{1}{2}\left\{\left(\Lambda_{1}+\Lambda_{2}\right)(1-m) a+\Lambda_{2}\left(\Lambda_{1}-\Lambda_{2}\right)(1-m) b\right. \\
& \left.+\left(\Lambda_{2}-\Lambda_{1}\right) m(m-1) \lambda(\mathcal{H})\right\}+\sum_{r=1}^{2 n-m+1} \sum_{1 \leq i<j \leq m}\left[h_{i i}^{r} h_{j j}^{r}-\left(h_{i j}^{r}\right)^{2}\right] .
\end{aligned}
$$

On the other hand, we have

$$
\begin{aligned}
m^{2}\|\mathcal{H}\|^{2}= & \sum_{r=1}^{2 n-m+1}\left(\sum_{i=1}^{m} h_{i i}^{r}\right)^{2}=\frac{1}{m-1} \sum_{r=1}^{2 n-m+1} \sum_{1 \leq i<j \leq m}\left(h_{i i}^{r}-h_{j j}^{r}\right)^{2} \\
& +\frac{2 m}{m-1} \sum_{r=1}^{2 n-m+1} \sum_{1 \leq i<j \leq m} h_{i i}^{r} h_{j j}^{r} .
\end{aligned}
$$

Further, from [6], we have

$$
\begin{aligned}
& \sum_{r=1}^{2 n-m+1} \sum_{1 \leq i<j \leq m}\left(h_{i i}^{r}-h_{j j}^{r}\right)^{2}+2 m \sum_{r=1}^{2 n-m+1} \sum_{1 \leq i<j \leq m}\left(h_{i i}^{r}\right)^{2} \\
& \geq 2 m\left[\sum_{1 \leq r<s \leq 2 n-m+11 \leq i<j \leq m}\left(h_{j k}^{r} h_{i k}^{s}-h_{i k}^{r} h_{j k}^{s}\right)^{2}\right]^{\frac{1}{2}} .
\end{aligned}
$$

Now, combining (3.3), (3.12) and (3.14), we have

$$
m^{2}\|\mathcal{H}\|^{2}-m^{2} \rho_{\mathcal{M}} \geq \frac{2 m}{m-1} \sum_{r=1}^{2 n-m+1} \sum_{1 \leq i<j \leq m}\left[h_{i i}^{r} h_{j j}^{r}-\left(h_{i j}^{r}\right)^{2}\right] \text {. }
$$

Taking into account (3.2), (3.11) and (3.14), we obtain the required inequality. 
Finally, by investigating the equality case of (3.5), the equality sign holds in (3.5) at a point $p \in \mathcal{M}$ if and only if the shape operators take the forms (3.6)-(3.8) with respect to some suitable tangent and normal orthonormal bases.

An immediate consequence of Theorem 3.1 yields the following:

Corollary 3.2. Let $\mathcal{M}$ be a minimalm-dimensional bi-slant submanifold in conformal Sasakian space form $\tilde{\mathcal{M}}(c)$ of dimension $(2 n+1)$ endowed with a quarter-symmetric connection, then we have

$$
\begin{aligned}
\rho_{\mathcal{M}}+\rho & \leq \exp (f)\left\{\frac{c+3}{4}-\frac{c-1}{2 m}+\frac{3(c-1)}{4 m(m-1)}\left(m_{1} \cos ^{2} \theta_{1}+m_{2} \cos ^{2} \theta_{2}\right)\right\}+\frac{\operatorname{tr}(B)}{m} \\
& +\frac{1}{4}\|\omega\|^{2}-\left\{\left(\Lambda_{1}+\Lambda_{2}\right) \frac{a}{m}+\Lambda_{2}\left(\Lambda_{1}-\Lambda_{2}\right) \frac{b}{m}-\left(\Lambda_{2}-\Lambda_{1}\right) \lambda(\mathcal{H})\right\} .
\end{aligned}
$$

Corollary 3.3. Let $\mathcal{M}$ be an m-dimensional submanifold in conformal Sasakian space form $\tilde{\mathcal{M}}(c)$ of dimension $(2 n+1)$ endowed with a quarter-symmetric connection, then we have Table 2:

For the semi-symmetric metric connection $\Lambda_{1}=\Lambda_{2}=1$, we have

Theorem 3.4. Let $\mathcal{M}$ be an m-dimensional bi-slant submanifold in conformal Sasakian space form $\tilde{\mathcal{M}}(c)$ of dimension $(2 n+1)$ endowed with a semi-symmetric metric connection, then we have

$$
\begin{aligned}
\rho_{\mathcal{M}}+\rho & \leq\|\mathcal{H}\|^{2}+\exp (f)\left\{\frac{c+3}{4}-\frac{c-1}{2 m}+\frac{3(c-1)}{4 m(m-1)}\left(m_{1} \cos ^{2} \theta_{1}+m_{2} \cos ^{2} \theta_{2}\right)\right\}+\frac{\operatorname{tr}(B)}{m} \\
& +\frac{1}{4}\|\omega\|^{2}-\frac{2 a}{m} .
\end{aligned}
$$

Corollary 3.5. Let $\mathcal{M}$ be an m-dimensional submanifold in conformal Sasakian space form $\tilde{\mathcal{M}}(c)$ of dimension $(2 n+1)$ endowed with a semi-symmetric metric connection, then we have Table 3:

For the semi-symmetric non-metric connection $\Lambda_{1}=1$ and $\Lambda_{2}=0$, we have

Theorem 3.6. Let $\mathcal{M}$ be an m-dimensional bi-slant submanifold in conformal Sasakian space form $\tilde{\mathcal{M}}(c)$ of dimension $(2 n+1)$ endowed with a semi-symmetric non-metric connection, then we have

$$
\begin{aligned}
\rho_{\mathcal{M}}+\rho & \leq\|\mathcal{H}\|^{2}+\exp (f)\left\{\frac{c+3}{4}-\frac{c-1}{2 m}+\frac{3(c-1)}{4 m(m-1)}\left(m_{1} \cos ^{2} \theta_{1}+m_{2} \cos ^{2} \theta_{2}\right)\right\} \\
& +\frac{\operatorname{tr}(B)}{m}+\frac{1}{4}\|\omega\|^{2}-\frac{a}{m}-\lambda(\mathcal{H}) .
\end{aligned}
$$

Corollary 3.7. Let $\mathcal{M}$ be an m-dimensional submanifold in conformal Sasakian space form $\tilde{\mathcal{M}}(c)$ of dimension $(2 n+1)$ endowed with a semi-symmetric non-metric connection, then we have Table 4 as follows: 
AJMS

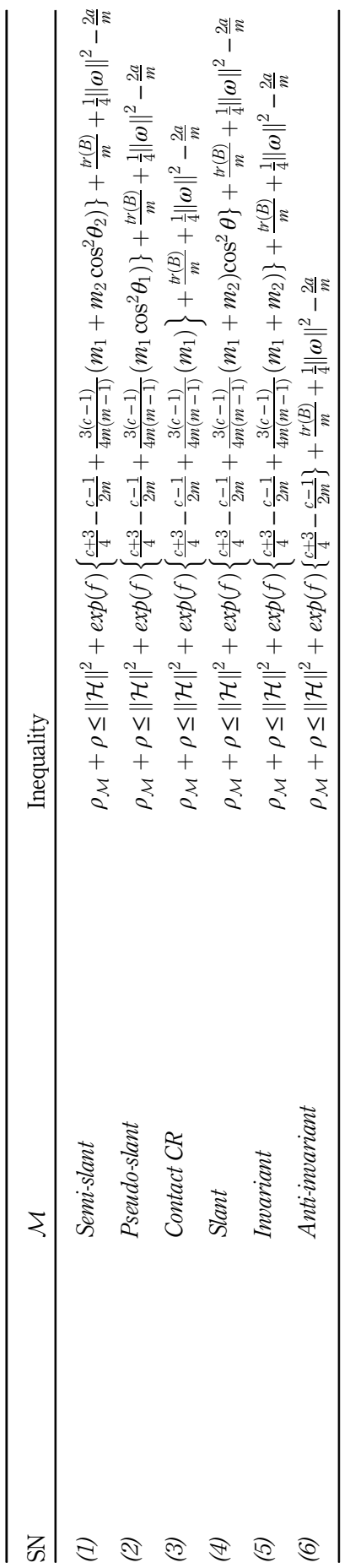

Table 3.

Inequalities for

different submanifolds

in a conformal

Sasakian space form

endowed with a semi-

symmetric metric

connection

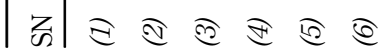




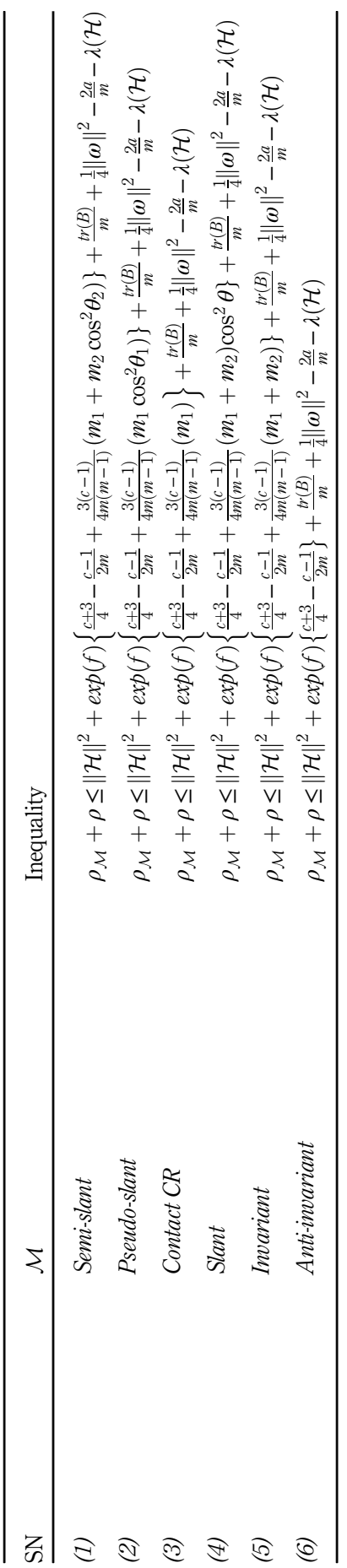

Generalized

Wintgen inequality

Table 4.

Inequalities for different submanifolds in a conformal Sasakian space form endowed with a semisymmetric non-metric connection 
In this segment, we provide some examples of a conformal Sasakian manifolds which is not Sasakian.

Example 4.1. Let us consider a three-dimensional manifold

$$
\tilde{\mathcal{M}}=\left\{\left(x_{1}, x_{2}, x_{3}\right) \in \mathbb{R}^{3}: x_{3}>0\right\},
$$

where $\left(x_{1}, x_{2}, x_{3}\right)$ are standard coordinates in $\mathbb{R}^{3}$. We choose the vector fields

$$
v_{1}=2\left(\frac{\partial}{\partial x_{1}}+x_{2} \frac{\partial}{\partial x_{3}}\right), v_{2}=2 \frac{\partial}{\partial x_{2}}, v_{3}=2\left(e^{x_{3} / 2}\right) \frac{\partial}{\partial x_{3}},
$$

which are linearly independent at each point of $\tilde{\mathcal{M}}$. Let $g$ be the Riemannian metric defined by

$$
g\left(v_{i}, v_{j}\right)=0, i \neq j, i, j=1,2,3, \quad g\left(v_{1}, v_{1}\right)=g\left(v_{2}, v_{2}\right)=e^{-x_{3}}, g\left(v_{3}, v_{3}\right)=1 .
$$

Let $\eta$ be the an 1-form defined by

$$
\eta\left(v_{3}\right)=1, \quad \eta\left(v_{2}\right)=0, \quad \eta\left(v_{1}\right)=0, \quad \eta(U)=g\left(U, v_{3}\right), \quad \forall U \in \Gamma(T \tilde{\mathcal{M}}) .
$$

We define the $(1,1)$ tensor field $\varphi$ as

$$
\varphi\left(v_{1}\right)=v_{2}, \quad \varphi\left(v_{2}\right)=-v_{1}, \quad \varphi\left(v_{3}\right)=0 .
$$

The linear property of $g$ and $\varphi$ yield that

$$
\begin{aligned}
& \eta\left(v_{3}\right)=1, \quad \varphi^{2}(U)=-U+\eta(U) v_{3} \\
& g(\varphi U, \varphi V)=g(U, V)-\eta(U) \eta(V),
\end{aligned}
$$

for any $U, V \in \Gamma(\tilde{\mathcal{M}})$. Thus, $(\tilde{\mathcal{M}}, \varphi, \xi, \eta, g)$ defines an almost contact metric manifold with $\xi=v_{3}$ [24]. Then we have

$$
\left[v_{1}, v_{2}\right]=-2 e^{-z^{1 / 2}}
$$

Similarly,

$$
\left[v_{1}, \xi\right]=x_{2} v_{3}, \quad\left[v_{2}, v_{3}\right]=0 .
$$

The Riemannian connection $\bar{\nabla}$ of the metric $g$ is given by

$$
\begin{gathered}
2 g\left(\bar{\nabla}_{X} Y, Z\right)=X g(Y, Z)+Y g(Z, X)-Z g(X, Y) \\
-g(X,[Y, Z])-g(Y,[X, Z])+g(Z,[X, Y]) .
\end{gathered}
$$

By Koszul's formula, we obtain the following

$$
\begin{gathered}
\bar{\nabla}_{v_{1}} v_{2}=-x_{3} v_{2}-e^{-x_{3}{ }^{1 / 2}} v_{3}, \quad \bar{\nabla}_{v_{1}} v_{3}=-e^{x_{3}{ }^{1 / 2}}\left(v_{1}-v_{2}\right), \quad \bar{\nabla}_{v_{1}} v_{1}=-x_{2} v_{1} e^{-x_{3}{ }^{1 / 2}} v_{3}, \\
\bar{\nabla}_{v_{2}} v_{1}=-x_{2} v_{2} e^{-x_{3}{ }^{1 / 2}} v_{3}, \quad \bar{\nabla}_{v_{2}} v_{2}=x_{2} v_{1} e^{-x_{3}{ }^{1 / 2}} v_{3}, \quad \bar{\nabla}_{v_{2}} v_{3}=-e^{-x_{3}{ }^{1 / 2}}\left(v_{1}+v_{2}\right), \\
\bar{\nabla}_{v_{3}} v_{3}=x_{2} v_{1} e^{x_{3}} v_{1}, \quad \bar{\nabla}_{v_{3}} v_{2}=x_{2} v_{1}+e^{-x_{3}{ }^{1 / 2}} v_{3}, \quad \bar{\nabla}_{v_{3}} v_{1}=-e^{x_{3}{ }^{1 / 2}}\left(v_{1}-v_{2}\right)-x_{2} v_{3} .
\end{gathered}
$$


Using contact transformation

$$
\tilde{\varphi}=\varphi, \quad \tilde{\xi}=e^{-x_{1} / 2} \xi, \quad \tilde{\eta}=e^{x_{1} / 2} \eta, \quad g=e^{x_{1}} g .
$$

$(\tilde{\mathcal{M}}, \tilde{\varphi}, \tilde{\xi}, \tilde{\eta}, g)$ is Sasakian manifold. So $\tilde{\mathcal{M}}$ is a conformal Sasakian manifold but not Sasakian. Since by the definition, we have

$$
\bar{\nabla}_{v_{2}} v_{3} \neq \varphi v_{2}
$$

for any $v_{1}, v_{2} \in \Gamma(\tilde{\mathcal{M}})$ (for instance $\bar{\nabla}_{v_{2}} v_{3} \neq 0$ ). By using the above results, we can find the nonvanishing components of Riemannian curvature, Ricci curvature tensor and scalar curvature as follows:

$$
\begin{gathered}
\overline{\mathcal{R}}\left(v_{1}, v_{2}\right) v_{2}=-4 v_{1}+x_{2} e^{-x_{3}{ }^{1 / 2}} v_{3}, \\
\overline{\mathcal{R}}\left(v_{1}, v_{3}\right) v_{3}=-v_{1}+3 v_{2}+x_{2} e^{-x_{3}{ }^{1 / 2}} v_{3}, \quad \overline{\mathcal{R}}\left(v_{2}, v_{3}\right) v_{3}=e^{x_{3}{ }^{1 / 2}} v_{1}+e^{-x_{3}{ }^{1 / 2}} v_{2}
\end{gathered}
$$

In view of above expressions, we turn up the following:

$$
\overline{\mathcal{K}}\left(v_{1}, v_{2}\right)=-4 e^{x_{3}}, \quad \overline{\mathcal{K}}\left(v_{1}, v_{3}\right)=-1, \quad \overline{\mathcal{K}}\left(v_{2}, v_{3}\right)=e^{x_{3}}\left(1-x_{2}\right) .
$$

Note that the sectional curvature of manifold $\tilde{\mathcal{M}}$ with almost contact-metric structure $(\tilde{\mathcal{M}}, \tilde{\varphi}, \tilde{\xi}, \tilde{\eta}, g)$ is

$$
\overline{\mathcal{K}}\left(v_{1}, v_{2}\right)=-3, \quad \overline{\mathcal{K}}\left(v_{1}, v_{3}\right)=-1, \quad \overline{\mathcal{K}}\left(v_{2}, v_{3}\right)=-1 .
$$

Moreover, the non-vanishing components of Ricci curvature tensor, and scalar curvature are given by

$$
\begin{gathered}
\overline{\mathcal{R i c}}\left(v_{2}, v_{2}\right)=-4, \quad \overline{\mathcal{R} i c}\left(v_{3}, v_{3}\right)=-1+e^{-x_{3} / 2} . \\
\bar{\rho}=-5+e^{-x_{3} / 2} .
\end{gathered}
$$

Example 4.2 Let us consider a three-dimensional manifold

$$
\tilde{\mathcal{M}}=\left\{\left(x_{1}, x_{2}, x_{3}\right) \in \mathbb{R}^{3}: x_{3}>0\right\},
$$

where $\left(x_{1}, x_{2}, x_{3}\right)$ are standard coordinates in $\mathbb{R}^{3}$. We choose the vector fields

$$
v_{1}=x_{1} \frac{\partial}{\partial x_{3}}, \quad v_{2}=x_{1} \frac{\partial}{\partial x_{2}}, \quad v_{3}=-\left(e^{-x_{1} / 2}\right) x_{1} \frac{\partial}{\partial x_{1}},
$$

which are linearly independent at each point of $\tilde{\mathcal{M}}$. Let $g$ be the Riemannian metric defined by

$$
g\left(v_{i}, v_{j}\right)=0, i \neq j, \quad i, j=1,2,3, \quad g\left(v_{1}, v_{1}\right)=g\left(v_{2}, v_{2}\right)=e^{-x_{1}}, \quad g\left(v_{3}, v_{3}\right)=1 .
$$

Let $\eta$ be the an 1-form defined by

$$
\eta\left(v_{3}\right)=1, \quad \eta\left(v_{2}\right)=0, \quad \eta\left(v_{1}\right)=0, \quad \eta(U)=g\left(U, v_{3}\right), \quad \forall U \in \Gamma(T \tilde{\mathcal{M}}) .
$$

We define the $(1,1)$ tensor field $\varphi$ by

$$
\varphi\left(v_{1}\right)=v_{2}, \quad \varphi\left(v_{2}\right)=-v_{1}, \quad \varphi\left(v_{3}\right)=0 .
$$


The linear property of $g$ and $\varphi$ yield

$$
\begin{aligned}
& \eta\left(v_{3}\right)=1, \quad \varphi^{2}(U)=-U+\eta(U) v_{3}, \\
& g(\varphi U, \varphi V)=g(U, V)-\eta(U) \eta(V),
\end{aligned}
$$

for any $U, V \in \Gamma(\tilde{\mathcal{M}})$. Thus, $(\tilde{\mathcal{M}}, \varphi, \xi, \eta, g)$ defines an almost contact metric manifold with $\xi=v_{3}$. Then, we have

$$
\left[v_{1}, v_{2}\right]=0
$$

Similarly,

$$
\left[v_{1}, \xi\right]=e^{x_{1} / 2} v_{1}, \quad\left[v_{2}, v_{3}\right]=e^{x_{1} / 2} .
$$

The Riemannian connection $\bar{\nabla}$ of the metric $g$ is given by

$$
\begin{gathered}
2 g\left(\bar{\nabla}_{X} Y, Z\right)=X g(Y, Z)+Y g(Z, X)-Z g(X, Y) \\
-g(X,[Y, Z])-g(Y,[X, Z])+g(Z,[X, Y]) .
\end{gathered}
$$

By Koszul's formula, we obtain the following:

$$
\begin{gathered}
\bar{\nabla}_{v_{1}} v_{2}=0, \quad \bar{\nabla}_{v_{1}} v_{3}=\frac{1}{2} e^{x_{1} / 2}\left(x_{1}+2\right) v_{1}, \quad \bar{\nabla}_{v_{1}} v_{1}=-\frac{1}{2} e^{x_{1} / 2}\left(x_{1}+2\right) v_{3}, \\
\bar{\nabla}_{v_{2}} v_{1}=0, \quad \bar{\nabla}_{v_{2}} v_{2}=-\frac{1}{2} e^{-x_{1} / 2}\left(x_{1}+2\right) v_{3}, \quad \bar{\nabla}_{v_{2}} v_{3}=\frac{1}{2} e^{x_{1} / 2} x_{1} v_{2}, \\
\bar{\nabla}_{v_{3}} v_{3}=0, \quad \bar{\nabla}_{v_{3}} v_{2}=\frac{1}{2} e^{x_{1} / 2} x_{1} v_{2}, \quad \bar{\nabla}_{v_{3}} v_{1}=-\frac{1}{2} e^{x_{1} / 2} x_{1} v_{1} .
\end{gathered}
$$

Adopting contact transformation

$$
\tilde{\varphi}=\varphi, \quad \tilde{\xi}=e^{-x_{1} / 2} \xi, \quad \tilde{\eta}=e^{x_{1} / 2} \eta, \quad \tilde{g}=e^{x_{1}} g .
$$

$(\tilde{\mathcal{M}}, \tilde{\varphi}, \tilde{\xi}, \tilde{\eta}, \tilde{g})$ is Sasakian manifold. Therefore, $\tilde{\mathcal{M}}$ is a conformal Sasakian manifold but not Sasakian, since by the definition, we have

$$
\left(\bar{\nabla}_{v_{2}} \varphi\right) v_{1} \neq g\left(v_{1}, v_{2}\right) \xi-\eta\left(v_{2}\right) v_{1}
$$

for any $v_{1}, v_{2} \in \Gamma(\tilde{\mathcal{M}})$. By using the above results, we find the non-vanishing components of Riemannian curvature, Ricci curvature tensor and scalar curvature.

$$
\begin{gathered}
\overline{\mathcal{R}}\left(v_{1}, v_{2}\right) v_{2}=-\frac{1}{4}\left(x_{1}+2\right)^{2} v_{1}, \quad \overline{\mathcal{R}}\left(v_{2}, v_{3}\right) v_{3}=-e^{x_{1}} v_{2}, \quad \overline{\mathcal{R}}\left(v_{1}, v_{2}\right) v_{3}=0, \\
\overline{\mathcal{R}}\left(v_{1}, v_{3}\right) v_{3}=-e^{x_{1}} v_{1}, \quad \overline{\mathcal{R}}\left(v_{3}, v_{1}\right) v_{1}=-v_{3}, \quad \overline{\mathcal{R}}\left(v_{3}, v_{1}\right) v_{2}=0, \\
\overline{\mathcal{R}}\left(v_{2}, v_{1}\right) v_{1}=-\frac{1}{4}\left(x_{1}+2\right)^{2} v_{2}, \quad \overline{\mathcal{R}}\left(v_{3}, v_{2}\right) v_{2}=-v_{3} . \\
\overline{\mathcal{R} i c}\left(v_{1}, v_{1}\right)=\overline{\mathcal{R} i c}\left(v_{2}, v_{2}\right)=\frac{1}{4}\left(x_{1}+2\right)^{2} e^{-x_{1}}, \quad \overline{\mathcal{R} i c}\left(v_{3}, v_{3}\right)=2 e^{-2 x_{1}} . \\
\bar{\rho}=e^{-x_{1}\left[\frac{1}{2}\left(-x_{1}+2\right)^{2}+2 e^{-x_{1}}\right]} .
\end{gathered}
$$


Example 4.3. Let $\mathbb{R}^{2 n+1}$ endowed with an almost contact structure $(\varphi, \xi, \eta, g)$ given by [3]

Generalized

$g=e^{-2 t}\left\{\eta \otimes \eta+\frac{1}{4} \sum_{i=1}^{n}\left\{\left(d x^{i}\right)^{2}+\left(d y^{i}\right)^{2}\right\}\right\}, \xi=e^{t}\left\{2 \frac{\partial}{\partial z}\right\}$,

Wintgen

inequality

$$
\varphi\left(\sum_{i=1}^{n}\left(X^{i} \frac{\partial}{\partial x^{i}}+Y^{i} \frac{\partial}{\partial y^{i}}\right)+Z \frac{\partial}{\partial z}\right)=\sum_{i=1}^{n}\left(Y^{i} \frac{\partial}{\partial x^{i}}-X^{i}\right) \frac{\partial}{\partial y^{i}}+\sum_{i=1}^{n} Y^{i} y^{i} \frac{\partial}{\partial z^{2}}
$$

where $t=\frac{1}{2}\left\{\sum_{i=1}^{n}\left(x^{i}\right)^{2}+\left(y^{i}\right)^{2}+z^{2}\right\}$.

Then, $\left(\mathbb{R}^{2 n+1}, \varphi, \xi, \eta, g\right)$ is not Sasakian manifold, but $\left(\mathbb{R}^{2 n+1}, \tilde{\varphi}, \tilde{\xi}, \tilde{\eta}, \tilde{g}\right)$ is Sasakian space form with constant $\tilde{\varphi}$-sectional curvature, where

$$
\begin{gathered}
\tilde{\varphi}=\varphi, \quad \tilde{\xi}=2 \frac{\partial}{\partial z}, \quad \tilde{\eta}=\frac{1}{2}\left(d z-\sum_{i=1}^{n} y^{i} d x^{i}\right), \\
\tilde{g}=\left\{\eta \otimes \eta+\frac{1}{4} \sum_{i=1}^{n}\left\{\left(d x^{i}\right)^{2}+\left(d y^{i}\right)^{2}\right\}\right\} .
\end{gathered}
$$

Therefore, $(\tilde{\mathcal{M}}, \tilde{\varphi}, \tilde{\xi}, \tilde{\eta}, \tilde{g})$ is the conformal Sasakian space form so that $(\tilde{\mathcal{M}}, \tilde{\varphi}, \tilde{\xi}, \tilde{\eta}, \tilde{g})$ is Sasakian space form of constant $\tilde{\varphi}$-sectional curvature $c=-3$.

Example 4.4. In [25], it was shown that the warped product $\mathbb{R} \times{ }_{f} \mathbb{C}^{n}$ is a generalized Sasakian space form with

$$
f_{1}=-\frac{\left(f^{\prime}\right)^{2}}{f^{2}}, f_{2}=0, f_{3}=-\frac{\left(f^{\prime}\right)^{2}}{f^{2}}+\frac{f^{\prime \prime}}{f},
$$

where $f=f(t), t \in \mathbb{R}$ and $f$ denotes the derivative of $f$ with respect to $t$. If we choose $n=4$ and $f(t)=e^{t}$, then $\tilde{\mathcal{M}}$ is a five-dimensional conformal Sasakian space form.

Example 4.5. Let us consider a 11-dimensional manifold $\tilde{\mathcal{M}}=\left\{\left(x_{1}, \ldots, x_{10}, z\right) \in \mathbb{R}^{11}: z \neq 0\right\}$, where $\left(x_{1}, x_{2}, x_{3}, x_{4}, x_{5}, x_{6}, x_{7}, x_{8}, x_{9}, x_{10}, z\right)$ are standard coordinates in $\mathbb{R}^{11}$. We choose the vector fields $E_{i}=e^{-z} \frac{\partial}{\partial x_{i}}, \quad i=1,2, \ldots, 10, \quad E_{11}=e^{-z} \frac{\partial}{\partial z}$ which are linearly independent at each point of $\tilde{\mathcal{M}}$. We define $\tilde{g}$ by

$$
\tilde{g}=e^{2 z} G,
$$

where $G$ is the Euclidean metric on $\mathbb{R}^{11}$. Hence $\left\{E_{i}\right\}_{i=1,2, \ldots, 11}$ is an orthonormal basis of $\tilde{\mathcal{M}}$.

We consider an one-form $\tilde{\eta}$ defined by

$$
\tilde{\eta}=e^{z} d z, \quad \tilde{\eta}(X)=\tilde{g}\left(X, E_{11}\right), \quad \forall X \in \Gamma(T \tilde{\mathcal{M}}) .
$$

We define the $(1,1)$ tensor field $\tilde{\varphi}$ by

$$
\tilde{\varphi}\left\{\sum_{i=1}^{5}\left(x_{i} \frac{\partial}{\partial x_{i}}+x_{i+5} \frac{\partial}{\partial x_{i+5}}+z \frac{\partial}{\partial z}\right)\right\}=\sum_{i=1}^{5}\left(x_{i} \frac{\partial}{\partial x_{i+5}}-x_{i+5} \frac{\partial}{\partial x_{i}}\right) .
$$


Thus, we have

$$
\tilde{\varphi}\left(E_{i}\right)=E_{i+5}, \quad \tilde{\varphi}\left(E_{i+5}\right)=-E_{i}, \quad \tilde{\varphi}\left(E_{11}\right)=0, \quad 1 \leq i \leq 5 .
$$

The linear property of $g$ and $\tilde{\varphi}$ yield that

$$
\begin{gathered}
\tilde{\eta}\left(E_{11}\right)=1, \quad \tilde{\varphi}^{2}(X)=-X+\tilde{\eta}(X) E_{11} \\
\tilde{g}(\tilde{\varphi} X, \tilde{\varphi} Y)=\tilde{g}(X, Y)-\tilde{\eta}(X) \tilde{\eta}(Y),
\end{gathered}
$$

for any $X, Y \in \Gamma(T \tilde{\mathcal{M}})$. Thus, $(\tilde{\mathcal{M}}, \tilde{\varphi}, \tilde{\xi}, \tilde{\eta}, \tilde{g})$ defines an almost contact-metric manifold with $\tilde{\xi}=E_{11}$. Then, we have $\left[E_{1}, E_{2}\right]=0$. Similarly, $\left[E_{i}, \tilde{\xi}\right]=e^{-z} E_{i},\left[E_{i}, E_{j}\right]=0,1 \leq i \neq j \leq 10$.

$$
\begin{gathered}
2 \tilde{g}\left(\bar{\nabla}_{X} Y, Z\right)=X \tilde{g}(Y, Z)+Y \tilde{g}(Z, X)-Z \tilde{g}(X, Y) \\
-\tilde{g}(X,[Y, Z])-\tilde{g}(Y,[X, Z])+\tilde{g}(Z,[X, Y]) .
\end{gathered}
$$

By Koszul's formula, we obtain the equations as follows:

$$
\bar{\nabla}_{E_{i}} E_{i}=-e^{-z} \tilde{\xi}, \quad \bar{\nabla}_{\tilde{\xi}} \tilde{\xi}=0, \quad \bar{\nabla}_{\tilde{\xi}} E_{i}=0, \quad \bar{\nabla}_{E_{i}} \tilde{\xi}=e^{-z} E_{i}, \quad 1 \leq i \leq 10 .
$$

Thus, we see that $\tilde{\mathcal{M}}$ is the conformal Sasakian manifold.

Now, we define a submanifold $\mathcal{M}$ of $\tilde{\mathcal{M}}$ by the immersion $\gamma$ as follows:

$\gamma\left(u_{1}, u_{2}, u_{3}, u_{4}, u_{5}, u_{6}, u_{7}\right)=e^{-z}\left(u_{1}, u_{3}, 0, \frac{1}{\sqrt{2}} u_{5}, \frac{1}{\sqrt{2}} u_{6}, u_{2}, u_{4} \cos \theta, u_{4} \sin \theta, \frac{1}{\sqrt{2}} u_{5}, \frac{1}{\sqrt{2}} u_{6}, u_{7}\right)$

for $0<\theta<\frac{\pi}{2}$.

It is easy to check that tangent bundle $T \mathcal{M}=\operatorname{Span}\left\{X_{1}, X_{2}, X_{3}, X_{4}, X_{5}, X_{6}, X_{7}\right\}$, where

$$
\begin{gathered}
X_{1}=e^{-z} \frac{\partial}{\partial x_{1}}, X_{2}=e^{-z} \frac{\partial}{\partial x_{6}}, X_{3}=e^{-z} \frac{\partial}{\partial x_{2}}, X_{4}=e^{-z}\left\{\cos \theta \frac{\partial}{\partial x_{7}}+\sin \theta \frac{\partial}{\partial x_{8}}\right\} \\
X_{5}=e^{-z} \frac{1}{\sqrt{2}}\left\{\frac{\partial}{\partial x_{4}}+\frac{\partial}{\partial x_{9}}\right\}, X_{6}=e^{-z} \frac{1}{\sqrt{2}}\left\{\frac{\partial}{\partial x_{5}}+\frac{\partial}{\partial x_{10}}\right\}, X_{7}=e^{-z} \frac{\partial}{\partial z} .
\end{gathered}
$$

Using the almost contact structure $\varphi$, we obtain

$$
\begin{gathered}
\tilde{\varphi} X_{1}=e^{-z} \frac{\partial}{\partial x_{6}}, \tilde{\varphi} X_{2}=-e^{-z} \frac{\partial}{\partial x_{1}}, \quad \tilde{\varphi} X_{3}=e^{-z} \frac{\partial}{\partial x_{7}} \\
\tilde{\varphi} X_{4}=-e^{-z}\left\{\cos \theta \frac{\partial}{\partial x_{2}}+\sin \theta \frac{\partial}{\partial x_{3}}\right\}, \quad \tilde{\varphi} X_{5}=e^{-z} \frac{1}{\sqrt{2}}\left\{-\frac{\partial}{\partial x_{4}}+\frac{\partial}{\partial x_{9}}\right\} \\
\tilde{\varphi} X_{6}=e^{-z} \frac{1}{\sqrt{2}}\left\{-\frac{\partial}{\partial x_{5}}+\frac{\partial}{\partial x_{10}}\right\}, \tilde{\varphi} X_{7}=0 .
\end{gathered}
$$

If we consider the distributions as follows:

$$
D_{\theta_{1}}=\operatorname{Span}\left\{X_{1}, X_{2}\right\}, D_{\theta_{2}}=\operatorname{Span}\left\{X_{3}, X_{4}\right\}, \quad \xi=\operatorname{Span}\left\{X_{5}, X_{6}\right\} .
$$

Then, we have $T \mathcal{M}=D_{\theta_{1}} \oplus D_{\theta_{2}} \oplus\langle\xi\rangle$. By some computations, it can be verified that $\mathcal{M}$ is bislant submanifold of $\tilde{\mathcal{M}}$. 


\section{References}

1. Vaisman I. Conformal changes of almost contact metric structures, Geometry and Differential Geometry. Lect Notes Math. 1980; 792: 435-43.

2. Wintgen P. Sur l'inégalité de Chen-Willmore. C. R. Acad Sci Paris. 1979; 288: 993-5.

3. Abedi E, Bahrami R, Tirpathi MM. Ricci and Scalar curvatures of submanifolds of a conformal Sasakian space form. Archivum Mathematicum (BRNO) Tomus. 2016; 52: 113-30.

4. Guadalupe IV, Rodriguez L. Normal curvature of surfaces in space forms. Pacific J Math. 1983; 106: $95-103$

5. Smet D, Dillen PJ, Verstraelen F, Vrancken LL. A pointwise inequality in submanifold theory. Arch Math. 1999; 35: 115-28.

6. Lu Z. Normal scalar curvature conjecture and its applications. J Funct Anal. 2011; 261: 1284-308.

7. Ge J, Tang Z. A proof of the DDVV conjecture and its equality case. Pac J Math. 2009; 237(1): 87-95.

8. Mihai I. On the generalized Wintgen inequality for Lagrangian submanifolds in complex space form. Nonlinear Anal. 2014; 95: 714-20.

9. Aydin ME, Mihai A, Mihai I. Generalized Wintgen inequality for statistical submanifolds in statistical manifolds of constant curvature. Bull Math Sci. 2017; 7: 155-66.

10. Mihai I. On the generalized Wintgen inequality for Legendrian submanifolds in Sasakian space forms. Tohoku Math J. 2017; 69(1): 43-53.

11. Murathan C, Sahin B. A study of Wintgen like inequality for submanifolds in statistical warped product manifolds. J Geom. 2018; 109(30): 1-18.

12. Alodan $\mathrm{H}$, Chen BY, Deshmukh S, Vilcu GE. A generalized Wintgen inequality for quaternionic CR-submanifolds. RACSAM. 2020; 114-29.

13. Lee CW, Lee JW, Vilcu GE. Generalized Wintgen inequality for submanifolds in generalized ( $\kappa$, $\mu$ )-space forms. Quaest Math. 2021; 1-15. doi: 10.2989/16073606.2021.1884140.

14. Siddiqui AN, Ahmad K. Generalized Wintgen inequality for totally real submanifolds in LCSmanifolds. Balkan J Geom App. 2019; 24(2): 53-62.

15. Chen BY. Recent developments in wintgen inequality and wintgen ideal submanifolds. Inter Elec J Geom. 2021; 14(1): 6-45.

16. Blair DE. Contact manifolds in riem annian geometry, math. New York: Springer-Verlag; 1976; 509.

17. Wang Y. Chen inequalities for submanifolds of complex space forms and Sasakian space forms with quarter-symmetric connection. Int J Geomet Methods Mod Phys. 2019; 16.

18. Cabrerizo JL, Carriazo A, Fernandez LM. Slant submanifolds in Sasakian manifolds. Glasgow Math J. 2000; 42(1): 125-38.

19. Cabrerizo JL, Carriazo A, Fernandez LM, Fernandez M. Semi-slant submanifolds of a Sasakian manifold. Geometriae Dedicata. 1999; 78: 183-99.

20. Chen BY. Geometry of slant submanifolds. Belgium: Katholieke Universiteit Leuven; 1990.

21. Chen BY, Uddin S. Warped product pointwise bi-slant submanifolds of Kaehler manifolds. Publ Math Debrecen. 2018; 92(1-2): 183-99.

22. Uddin S, Chen BY, Al-Solamy FR. Warped product bi-slant immersions in Kaehler manifolds. Mediterr J Math. 2017; 14(2): 11. Art. 95.

23. Uddin S, Lone MS., Lone MA. Chen's $\delta$-invariants type inequalities for bi-slant submanifolds in generalized Sasakian space forms. J Geom Phys. 2021; 161: 104040.

24. Abedi E, Ilmakchi M. Submanifolds of a conformal Sasakian manifold. Bol Soc Paran Mat. 2021; 39(1): 23-34. 
25. Alegre P, Blair DE, Carriazo A. Generalized Sasakian space-forms. Isr J Math. 2004; 141: $157-83$.

Corresponding author

Aliya Naaz Siddiqui can be contacted at: aliyanaazsiddiqui9@gmail.com

For instructions on how to order reprints of this article, please visit our website: www.emeraldgrouppublishing.com/licensing/reprints.htm Or contact us for further details: permissions@emeraldinsight.com 